\title{
Overlapping PPM for band-limited visible light communication and dimming
}

\author{
John E. Gancarz, Hany Elgala* and Thomas D.C. Little
}

\author{
* Correspondence: helgala@bu.edu \\ Department of Electrical and \\ Computer Engineering, Multimedia \\ Communications Lab (MCL), NSF \\ Smart Lighting Engineering \\ Research Center, Boston University, \\ 8 Saint Mary's Street, Boston 02115 \\ MA, USA
}

\begin{abstract}
The synthesis of visible light communication (VLC) and lighting state control necessitates data-light modulation that can accommodate intensity control. A number of techniques that enable both optical wireless data transmission and intensity control of light-emitting diodes (LEDs) have been proposed as a response to this need. Relevant schemes leverage amplitude modulation (AM)/continuous current reduction (CCR) and/or pulse-width modulation (PWM) for dimming capability. Two-level schemes related to PWM, such as on-off keying with compensation time $(\mathrm{OOK}+\mathrm{CT})$, variable pulse position modulation (VPPM), and multiple pulse position modulation (MPPM), are most commonly investigated. In this paper, we survey and compare OOK $+C T$, VPPM and MPPM. Moreover, we propose a novel approach towards dimming and data transmission through the variation of codeword weights in overlapping pulse-position modulation (OPPM). The proposed approach has comparatively high spectral efficiency. Using realistic constraints of a practical VLC system, analysis reveals that OPPM can increase data rates by more than 20Mbps over expected performance of related, two-level schemes, when using LEDs suitable for lighting that have relatively low modulation bandwidths.

Keywords: Dimming; IEEE 802.15.7 standard; Light-emitting diode (LED); Lighting control; Optical wireless communication (OWC); Overlapping pulse-position modulation (OPPM); Visible light communication (VLC)
\end{abstract}

\section{Background}

Future lighting systems will be expected to be optimized to meet strict energy efficiency and light rendering quality goals. To be commercially competitive, they will increasingly have new functions providing adaptability, self-provisioning capabilities, and intelligence to react to human needs. These functions will be provided by embedded control and communications. Visible light communications (VLC) seeks to provide high-speed optical communications as an additional function, delivering additional capacity in indoor wireless networks. Although these functions leverage one another (embedded control, communications, and VLC), the common lighting task of intensity control (dimming) is in conflict with achieving optical data modulation. VLC systems, which stream data wirelessly by high frequency modulation of LED drive currents, must incorporate new modulation schemes for compatibility with lighting control [1].

(c) 2015 Gancarz et al. This is an Open Access article distributed under the terms of the Creative Commons Attribution License (http:// creativecommons.org/licenses/by/4.0), which permits unrestricted use, distribution, and reproduction in any medium, provided the original work is properly credited. 
These usually result in increased complexity and decreases in performance as compared to systems designed for fixed lighting conditions. Dimming schemes inherently limit the transmitted optical power and place restrictions on the modulated waveform. Additionally, reducing light intensity can result in an undesired chromaticity shift of the emitted light [2]. While a number of modulation schemes compatible with dimming requirements have been proposed to mitigate data rate and light quality losses, as described in [2-5], analysis of schemes from a comprehensive perspective is often lacking. Consequently, this paper seeks to incorporate realistic constraints, including the limited bandwidths of LEDs, in evaluating two-level baseband data modulation and dimming schemes.

We propose a novel operation through the variation of codeword weights of overlapping pulse position modulation (OPPM) to achieve dynamic dimming and data transmission at higher data rates compared to well-known common two-level modulation and dimming schemes. For noise-limited environments, other schemes may yield higher data rates than OPPM; yet, the subsequent analysis reveals that within a bandlimited regime, the higher spectral efficiency of OPPM results in significant data rate gains over comparable schemes.

In this paper, we review the VLC channel model, followed by an overview of common two-level modulation and dimming, a description of OPPM and its dimming functionality, and a presentation of the analysis revealing the advantages of OPPM. The final section concludes the findings.

\section{Methods}

\section{VLC channel model}

The basic optical wireless channel model adopted for VLC is of the form,

$$
y(t)=r \cdot x(t) * h(t)+n(t),
$$

where $r$ is the responsivity of the photodiode receiver [A/W], $x(t)$ is instantaneous optical power, $h(t)$ is the channel impulse response, $n(t)$ is added noise, and * denotes convolution. For VLC, intensity modulation with direct detection (IM/DD) is nearly always adopted, whereby the instantaneous optical power emitted by the LED transmitter is fluctuated for data transmission. One of the ramifications of IM/DD is that $x(t)$ is always greater than or equal to zero (optical power is always a positive value). The average received power, $P_{n}$ is defined as follows for optical wireless systems,

$$
P_{r}=H(0) P_{t}=\left(\int_{-\infty}^{\infty} h(t) d t\right)\left(\lim _{T \rightarrow \infty} \frac{1}{2 T} \int_{-T}^{T} x(t) d t\right)
$$

where $H(0)$ is the DC channel gain calculated from the impulse response $h(t), T$ defines the integral limit for the time average of $\mathrm{x}(\mathrm{t})$, and $P_{t}$ is the transmitted power [6].

VLC shares considerable characteristics and challenges with wireless infrared (IR) communications. Equations (1) and (2) are equally applicable to VLC from IR communication theory, as well as the basic signal-to-noise ratio (SNR) [6], 


$$
S N R=\frac{r^{2} H^{2}(0) P_{t}^{2}}{R_{b} N_{0}}=\frac{r^{2} P_{r}^{2}}{R_{b} N_{0}}
$$

where $N_{O}$ is the double-sided power spectral density of white Gaussian noise (assuming this noise is dominant) and $\mathrm{R}_{\mathrm{b}}$ is the bit-rate. For the model environment considered in the analysis, it is assumed that inter-symbol interference (ISI) is negligible [7].

\section{Dimming and two-level modulation techniques}

The two most popular methods of dimming LEDs are amplitude modulation (AM)/ continuous current reduction (CCR) and pulse-width modulation (PWM) (see Fig. 1). $\mathrm{AM}$, also known as analog dimming, reduces the LED drive current to lessen the brightness of emitted light. PWM cycles the LED with high and low drive currents, alternating between on and off states.

AM dimming has a few significant drawbacks. AM may induce a noticeable chromaticity shift in the emitted light, i.e. the light color will change as the LED is dimmed, especially if dimming at low light levels. Additionally, AM dimming is nonlinear; a change in the forward current is not directly proportional to the luminance of the emitted light. Conversely, PWM generally does not induce a perceptible chromaticity shift and features a near-linear relationship between luminance and duty cycle. Dyble et al. demonstrated the superior color integrity properties of PWM versus AM in their evaluation of white LEDs and dimming [2].

A number of two-level baseband communication and dimming schemes are similar to PWM in that they have two states (high and low) and control brightness by varying the average time duration of the high state to the low state. This paper concentrates on these schemes as they stand to inherit the advantageous color integrity and linearity characteristics of PWM dimming. For comparative analysis, maximum-likelihood (ML) detection and high-SNR is assumed. In this case, the bit-error ratio (BER) is principally influenced by the two nearest, modulated signals in which,

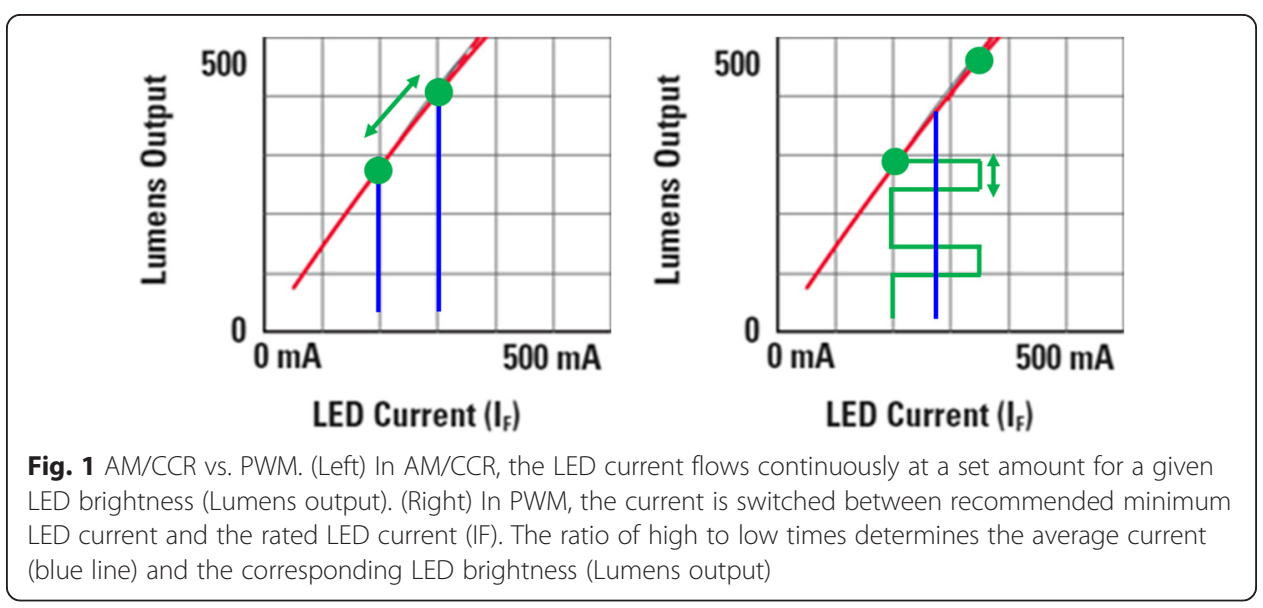




$$
B E R \approx Q\left(\frac{\sqrt{\min _{i \neq j} \int\left(x_{i}(t)-x_{j}(t)\right)^{2} d t}}{2 \sqrt{N_{0}}}\right)=Q\left(\frac{d_{\text {min }}}{2 \sqrt{N_{0}}}\right),
$$

where $d_{\text {min }}$ is the minimum Euclidean distance between signals within the valid signal set. Park, Barry, and Lee [3, 8] also adopt this approach for the additive white Gaussian noise (AWGN), IM/DD channel. An overview of two-level schemes for simultaneous communication and dimming is contained within the following subsections.

\section{OOK with compensation time}

Unipolar, non-return-to-zero on-off keying (OOK) is the simplest IM/DD communication scheme (see Fig. 2). On (high level) denotes a one bit and off (low level) denotes a zero bit. One method of guaranteeing a DC balanced signal, where the average value of the signal is equally between the low and high luminance levels, is Manchester line coding; a 0-bit is a low-high transition and a 1-bit is a high-low transition. Thus, OOK with Manchester line coding has an average power that is $50 \%$ of the peak power and, correspondingly, the emitted light is $50 \%$ of the maximum LED luminance. Its minimum Euclidean distance is given in (5),

$$
d_{\mathrm{min}, \mathrm{OOK}}=\frac{2 P_{\text {avg }}}{\sqrt{R_{b}}}=\frac{P_{p k}}{\sqrt{R_{b}}}
$$

where the average power $P_{a v g}$ is half of the peak power $P_{p k}$. It is assumed that the bandwidth of OOK is approximately equal to $R_{b}$ [8].

OOK with compensation time $(\mathrm{OOK}+\mathrm{CT})$ consists of a data subframe followed or preceded by a CT subframe. CT is utilized to raise or lower average power of a frame to brighten or dim. The CT subframe is fixed at a high or low level, i.e. the LED is

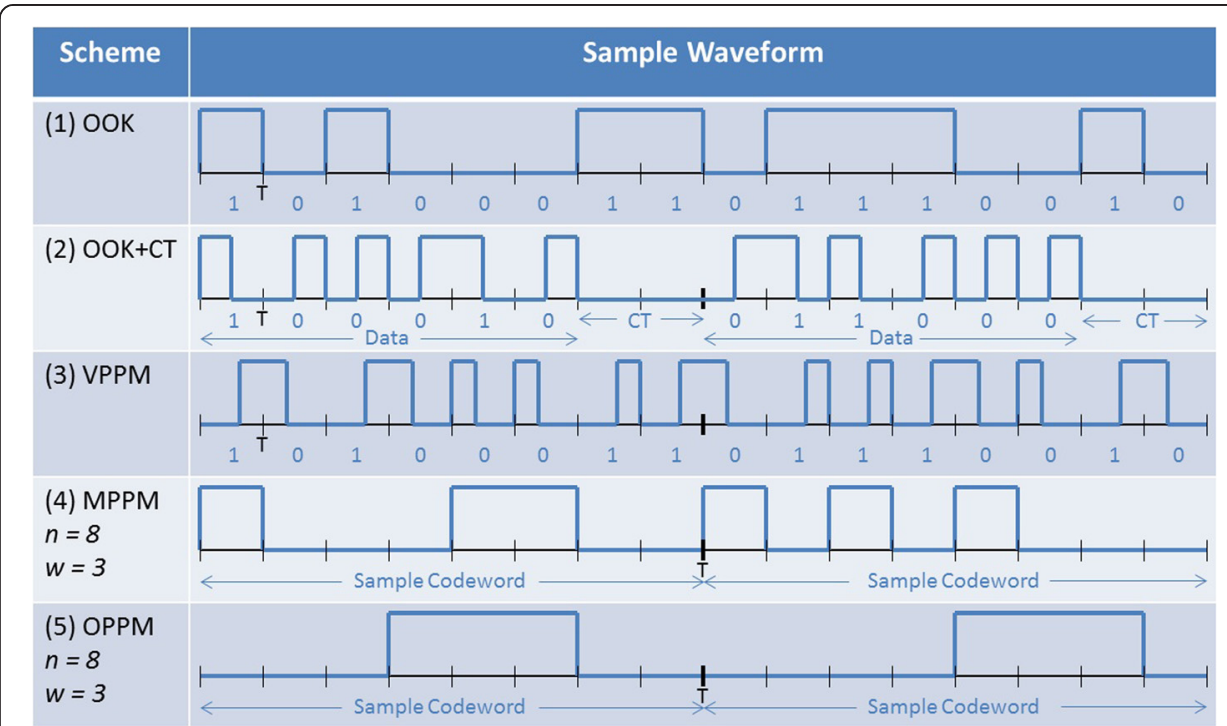

Fig. 2 Two-level modulation and dimming schemes to obtain about $60 \%$ dimming [1]. (1) OOK: considered as reference at $50 \%$ dimming, (2) OOK + CT using Manchester line coding, (3) VPPM: pulse width of conventional 2-PPM is reduced from 1/2 to 3/8, (4) MPPM: 56 possible codewords, and (5) OPPM: 6 possible codewords 
completely driven to its on state or off state, depending on whether one wishes to brighten above $50 \%$ or dim below $50 \%$. The duration of the CT determines the precise proportion of peak power, $p$, that is emitted $\left(p=P_{\text {avg }} / P_{p k}\right)$.

Equations (4) and (5) yield the following theoretically achievable bit-rate for OOK+ CT and a given $p$,

$$
R_{b}=\left\{\begin{array}{ll}
\left(\frac{P_{p k}}{Q^{-1}(B E R)}\right)^{2} \frac{p}{2 N_{0}}, & 0<p<0.5 \\
\left(\frac{P_{p k}}{Q^{-1}(B E R)}\right)^{2} \frac{(1-p)}{2 N_{0}}, & 0.5 \leq p<1
\end{array} .\right.
$$

Note that the bit-rate is scaled compared to standard OOK due to the CT for dimming and brightening. Naturally, bit-errors are only considered for the data frames. For a given bandwidth requirement $B, R_{b}$ must satisfy,

$$
R_{b} \leq\left\{\begin{array}{cc}
p B, & 0<p<0.5 \\
(1-p) B, & 0.5 \leq p<1
\end{array} .\right.
$$

This takes into account the fact that Manchester line-coded OOK requires twice the bandwidth of standard OOK. OOK $+\mathrm{CT}$ as presented is the elementary version of the first physical type of IEEE 802.15.7 Visible Light Communication Task Group standard [4].

\section{Variable Pulse-Position Modulation (VPPM)}

VPPM is a modified version of 2-PPM, in which each symbol is divided into two slots, or chips (see Fig. 2). In classic 2-PPM, a 0-bit is communicated by a pulse in the first slot and a 1-bit is communicated by a pulse in the second slot. VPPM differs in that, for a fixed symbol duration, the pulses can shrink or expand in width to achieve the desired average power corresponding to the dimming set point. Performance necessarily decreases when deviating from 2-PPM (when $p=0.5$ ) due to a reduction in the Euclidean distance of the 0-bit and 1-bit symbols. VPPM has the same achievable $R_{b}$ and bandwidth constraint as $\mathrm{OOK}+\mathrm{CT}$ in (6) and (7). The technique is also a basic version of the second physical type of the IEEE 802.15.7 standard [4].

\section{Multiple Pulse-Position Modulation (MPPM)}

MPPM as a modulation technique for simultaneous communication and dimming was presented in [3]. MPPM is similar to classic L-PPM; however, instead of allowing for one pulse per symbol period in one of $L$ chips, it permits multiple pulses in any of the $L$ chips (see Fig. 2). Each unique combination of pulses within a symbol period is represented by a codeword. A possible codeword is $c_{1}=[0,1,1,0]$, representing a symbol where there is no pulse in chips 1 and 4, a pulse in chip 2, and a pulse in chip 3 . MPPM is characterized by two values, the number of chips per symbol, $n$, and the weight of accepted codewords, $w$. The weight, $w$, is equivalent to the sum of ones in a codeword. As in [3], we assume that the weight of codewords is fixed. Therefore, there are $Q=(n, w)$ possible codewords where $(n, w)$ is the binomial coefficient. Q is the alphabet size. $L$-PPM is a limiting case of MPPM where $w=1$ and $n=Q=L$. 
Lee and Park utilize MPPM to brighten or dim by varying the weight of the codewords [3]. For example, $(8,2)$ MPPM will result in the average power of transmission being $1 / 4^{\text {th }}$ of the peak power, $p=0.25$. The ratio $p$ is equivalent to $w / n$.

Equations (8) and (9) specify the achievable bit-rate for an AWGN channel and the bit-rate ceiling for a bandwidth limit $B$.

$$
\begin{aligned}
& R_{b} \approx\left(\frac{P_{p k}}{Q^{-1}(B E R)}\right)^{2} \frac{\log _{2}\left(\begin{array}{c}
n \\
w
\end{array}\right)}{2 n \cdot N_{0}} \\
& R_{b} \leq \frac{B \cdot \log _{2}\left(\begin{array}{l}
n \\
w
\end{array}\right)}{n}
\end{aligned}
$$

\section{Overlapping Pulse-Position Modulation (OPPM)}

OPPM follows directly from MPPM with the additional constraint that all pulses must be consecutive, i.e. all ones must be contiguous in a codeword (see Fig. 2). This extra specification necessitates a decrease in the number of permitted codewords; only $Q=n-w+1$ codewords exist as compared to $(n, w)$ for MPPM. The benefit of OPPM lies instead in spectral efficiency, which is greater than that of MPPM. This allows for a looser bound for $R_{b}$, given the bandwidth constraint, $B$. Building off the theory in [8], Equations (10) and (11) specify the achievable bit-rate and ceiling due to the bandwidth limit.

$$
\begin{aligned}
& R_{b} \approx\left(\frac{P_{p k}}{Q^{-1}(B E R)}\right)^{2} \frac{\log _{2}(n-w+1)}{2 n \cdot N_{0}} \\
& R_{b} \leq \frac{B \cdot w \cdot \log _{2}(n-w+1)}{n}
\end{aligned}
$$

As with MPPM, the value of $w$ can be changed to switch between intensity levels to match the dimming preferences specified by the user. This is illustrated in Fig. 3. In (10), it is evident that as $w$ approaches $n, R_{b}$ decreases. The bandwidth limitation of (11) takes into account the lower bandwidths of using longer pulses with the corresponding sacrifice of alphabet size.

Bo et al. discussed OPPM dimming in [5]; however, their approach utilizes AM dimming, i.e. the $P_{p k}$ is reduced to reduce light intensity. As explained in Section III, AM has certain

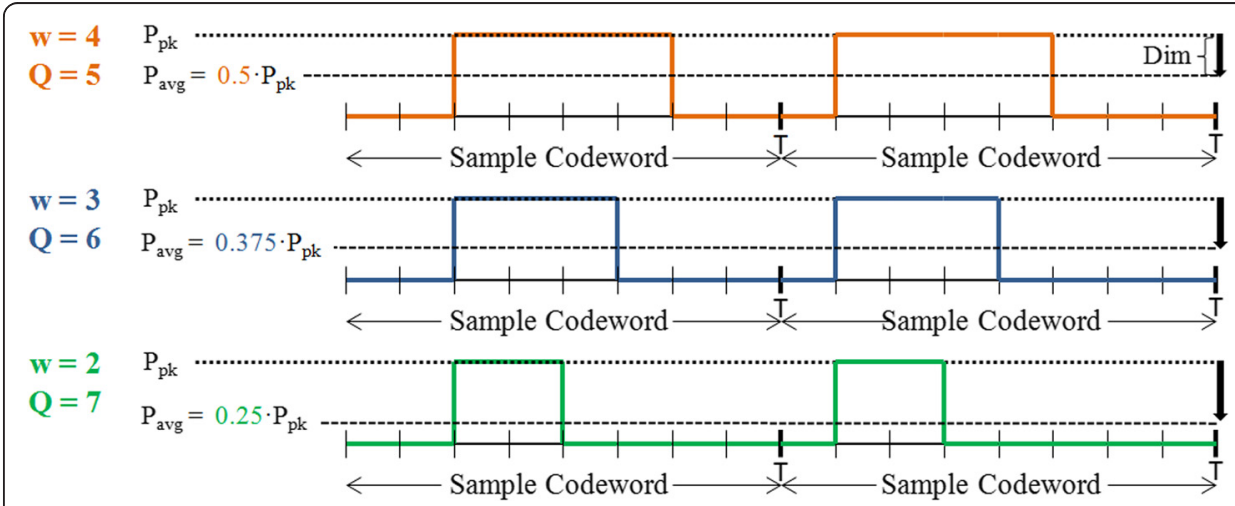

Fig. 3 OPPM dimming example. As the codeword weight, $w$, decreases, the $P_{\text {avg }}$ decreases, which results in a reduction of light intensity as perceived by the human eye. The number of valid codewords, $Q$, for a fixed $\mathrm{W}$ is $\mathrm{Q}=\mathrm{n}-\mathrm{W}+1$, where $\mathrm{n}$ is the number of chips per symbol ( $n=8$ in this example) 
disadvantages with regard to light quality. Nonetheless, reference [5] demonstrates that OPPM with AM dimming fares well considering a flicker severity index as compared to OOK and VPPM. Future research is necessary to conclude if a hybrid of OPPM dimming using AM and the varying of codeword weights may inherit benefits of both techniques.

\section{Results and discussion}

The analysis aims at incorporating realistic constraints of a practical VLC system. As opposed to the traditional BER versus SNR comparison, we investigate achievable bit-rate versus perceived brightness level. This approach encompasses the fixed peak power limit of artificial lighting in a given room. A BER of $10^{-6}$ is targeted. Perceived brightness is calculated as,

$$
\text { Perceived Brightness }(\%)=100 \sqrt{\frac{P_{\text {avg }}}{P_{p k}}}=100 \sqrt{p} \text {. }
$$

Equation (12) is a form of Stevens' power law also presented in [4]. It accounts for the nonlinear response of the human visual system to changes in luminance.

As evident from (6)-(11), the bit-rate can be noise-limited or band-limited at a particular perceived brightness. As one dims or brightens, changing $p$, it is possible to transition from a noise-limited regime to a band-limited regime (while keeping the same BER). The particular behavior is dictated by the noise and/or signal strength.

For the sake of comparison, a $5 \times 5 \times 3 \mathrm{~m}^{3}$ model room is studied having the same geometry and transmitter/receiver properties as scenario $B$ of Grubor et al.'s work in reference [7]. This configuration requires that $80 \%$ of the illuminance at desktop level ( $0.85 \mathrm{~m}$ from floor) is above 400 lux, a typical requirement for office lighting. Four $1 \times 1$ $\mathrm{m}^{2}$ luminaires in the model room each contain 196 LED chips capable of emitting $12.3 \mathrm{~W}$ of radiant flux. A photodiode with responsivity $0.28 \mathrm{~A} / \mathrm{W}$ is assumed as per conformity with [7].

In order to demonstrate that the bandwidth limitation is dominant even in dimly lit regions of the model room, the minimum recorded SNR, $47 \mathrm{~dB}$, and received power, $0.1 \mathrm{~mW}$, were adopted from the simulation of Grubor et al. The LEDs are at fullbrightness for the SNR calculation, but certain regions of the room receive more light than others due the lighting configuration and reflections.

$N_{O}$ can be calculated to be approximately $7.82 \times 10^{-22} \mathrm{~A}^{2} / \mathrm{Hz}$ using (3) and substituting $R_{b}$ for a bandwidth of $20 \mathrm{MHz}$, as defined in [7]. A bandwidth of $20 \mathrm{MHz}$ is approximately the maximum value for phosphor-converted, white LEDs used in conjunction with a blue-filter at the receiver. The theoretically achievable bit-rate can be seen in Fig. 4 for OOK + CT, VPPM, MPPM $(n=32, n=8)$, and OPPM $(n=32, n=8)$. Note that $\mathrm{OOK}+\mathrm{CT}$ and VPPM are, in theory, continuous dimming schemes, whereas the others are discrete. A large number of chips can approximate continuous dimming [3]. Excluding the extremes (near $0 \%$ and $100 \%$ ), the schemes are band-limited across all perceived brightness levels, i.e. the LED bandwidth is the bottleneck of communication rather than noise. OPPM proves to be superior to the other dimming and data communication schemes.

As shown in Fig. 4, OPPM $(n=32)$ achieves 40.9 Mbps at a perceived brightness of $70.7 \%$, which is $22.6 \mathrm{Mbps}$ greater than MPPM $(n=32)$ and 30.9 Mbps greater than 


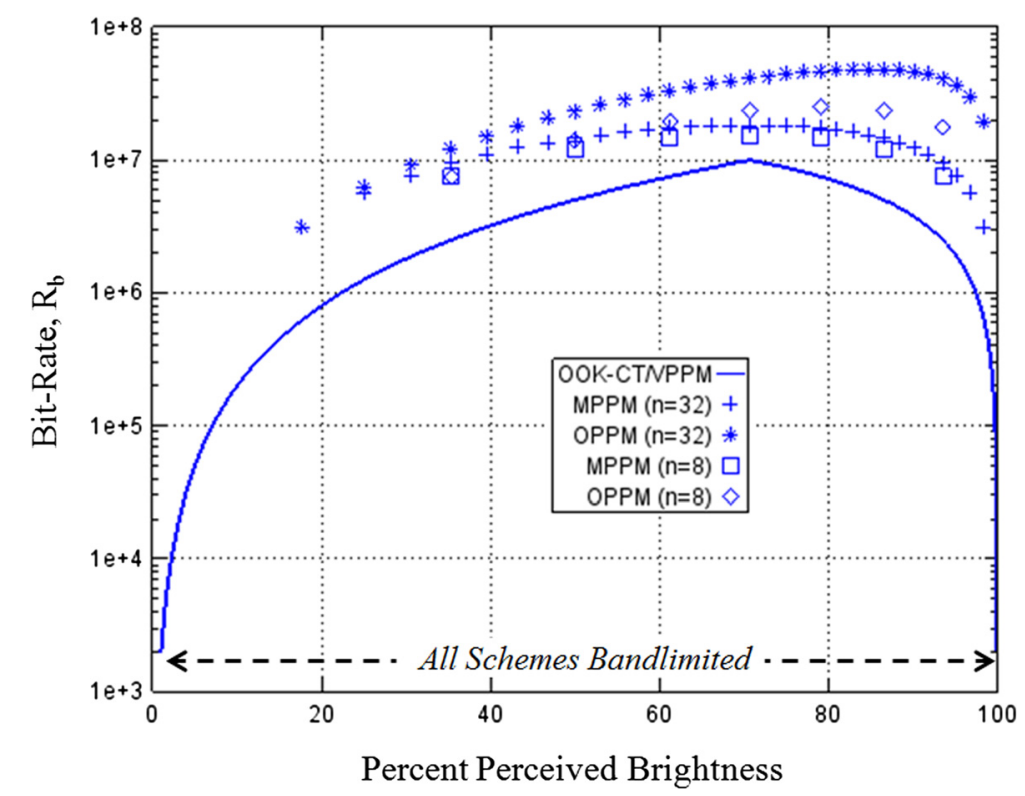

Fig. 4 Achievable bit-rate versus perceived brightness level when $N_{0}=7.82 \times 10^{-22} \mathrm{~A}^{2} / \mathrm{Hz}(r=0.28 \mathrm{~A} / \mathrm{W}$, $P_{p k}=0.1 \mathrm{~mW}, B E R=10^{-6}, B=20 \mathrm{MHz}$ ). The superior performance of OPPM in this regime is evident. All schemes are band-limited across the entire dimming range

OOK-CT/VPPM. If brightness is increased to $98.4 \%$ (31/32 of peak power), OPPM $(n=32)$ drops to a bit rate of 19.4 Mbps, but still maintains superiority by achieving $16.3 \mathrm{Mbps}$ over MPPM $(n=32)$ and $18.8 \mathrm{Mbps}$ over OOK-CT/VPPM. Dimming to $17.7 \%$ (1/32 of peak power), MPPM matches OPPM at a bit-rate of $3.1 \mathrm{Mbps}$, which is $2.5 \mathrm{Mbps}$ greater than the bit-rate achieved by OOK + CT/VPPM. MPPM and OPPM are essentially both $32-\mathrm{PPM}$ at this percentage (since $w=1$ ).

\section{Conclusions}

Dimming, or intensity control, is the most prevalent and popular form of lighting control. In addition to creating the desired ambience, dimming can also provide energy savings. Unsurprisingly however, dimming presents a number of immediate challenges to VLC as modulation schemes must enable both data transmission and light intensity adjustment.

In this paper, we proposed OPPM dimming, wherein the weight of codewords is modified according to the desired dimming percentage. The technique manifests its usefulness in a comprehensive analysis of a VLC environment, which takes into account LED bandwidth limitations. Analysis reveals gains of over $20 \mathrm{Mbps}$ in using OPPM rather than other two-level schemes in band-limited environments. Noise levels must increase by over two orders of magnitude than the model case considered in the analysis in order for MPPM with the same number of chips to surpass the performance of OPPM. 


\section{Authors' contributions}

TL proposed the topic, conceived and designed the study. JG carried out the study. HE analyzed the data, helped in their interpretation and collaborated with JG in the construction of manuscript. All authors read and approved the final manuscript.

\section{Authors' information}

John Gancarz received his B.S. and M.S. in electrical engineering from Boston University in 2011 and 2013, respectively. His prior research work was in the field of electromagnetics and plasma science. Particularly, he studied local geomagnetic field fluctuations induced by electromagnetic heater waves injected into ionospheric plasma. In 2011, he joined the NSF Smart Lighting ERC at Boston University. His initial research concerned novel light rendering techniques in "smart" lighting systems. Additional research interests were VLC related, including adaptive data rate and coding, illumination quality effects of VLC, and the synthesis of modulation and dimming schemes. He is commencing priestly studies and formation at the Pontifical North American College and the Pontifical Gregorian University in Rome, Italy.

Hany Elgala is a Research Professor in the Department of Electrical \& Computer Engineering at Boston University. Hany is a member of the Multimedia Communications Lab (MCL) and the NSF Smart Lighting Engineering Research Center (ERC). From 2010-12, Dr. Elgala was the co-leader of the Cellular and Wireless Communications (CWC) Lab at Jacobs University in Germany. There, he coordinated two industrial projects with Airbus Germany and the European Aeronautic Defence and Space Company (EADS) to realize high-speed optical wireless networks in airplane cabins. Hany received the B.Sc. in Electronics and Communications from Ain-Shams University in 2000, the M.Sc. in Microsystems Engineering from Furtwangen University in 2003, and Ph.D. in Optical Wireless Communications from Jacobs University in 2010. He is the author or co-author of approximately 40 publications in the area of optical wireless communications. His research focuses on wireless communications and networking, visible light communications, OFDM systems and MIMO transmission. Thomas D.C. Little is a professor in the Department of Electrical and Computer Engineering at Boston University. He is Associate Dean of Educational Initiatives for the College and Associate Director of the NSF Smart Lighting Engineering Research Center - a collaboration of Rensselaer Polytechnic Institute, the University of New Mexico, and Boston University. Recent efforts include research in video sensor networks and streaming in wireless settings, ubiquitous optical networking with visible light, vehicle-to-vehicle/infrastructure (V2X) communications, and the application of wireless sensors in health monitoring. Dr. Little received the BS degree in biomedical engineering from Rensselaer Polytechnic Institute in 1983, and the MS degree in electrical engineering and PhD degree in computer engineering from Syracuse University in 1989 and 1991. He is a senior member of the IEEE, a member of the IEEE Computer and Communications Societies and a member of the Association for Computing Machinery.

\section{Acknowledgements}

This work is supported by the National Science Foundation under Grant No. EEC-0812056.

Received: 22 December 2014 Accepted: 5 May 2015

Published online: 27 May 2015

\section{References}

1. Gancarz J, Elgala H, Little TDC. Impact of lighting requirements on VLC systems. IEEE Commun Mag. 2013:51(12):34-41.

2. Dyble M, Narendran N, Bierman A, Klein T. Impact of dimming white LEDs: chromaticity shifts due to different dimming methods. Proc. SPIE 5941, Fifth International Conference on Solid State Lighting, 59411H. 2005; doi10.1117/12.625924. http://proceedings.spiedigitallibrary.org/proceeding.aspx?articleid=873212\&resultClick=1.

3. Lee K, Park H. Modulations for Visible Light Communications with Dimming Control. IEEE Photon Technol Lett. 2011;23(16):1136-8

4. Rajagopal S, Roberts RD, Lim S. IEEE 802.15.7 visible light communication: modulation schemes and dimming support. IEEE Commun Mag. 2012;50(3):72-82.

5. Bo B, Zhengyuan $X$, Yangyu F. Joint LED dimming and high capacity visible light communication by overlapping PPM. 19th Annual Wireless and Optical Communications Conference (WOCC). 2010;1-5. doi:10.1109/WOCC.2010.5510410.

6. Kahn JM, Barry JR. Wireless infrared communications. Proceedings of the IEEE. 1997;85(2):265-98.

7. Grubor J, Jamett OC, Walewski JW, Randel S, Langer K-D. High-speed wireless indoor communication via visible light. ITG-Fachbericht-Breitbandversorgung in Deutschland-Vielfalt für alle? 2007.

8. Park H, Barry JR. Modulation analysis for wireless infrared communications. IEEE International Conference on Communications (ICC) (Gateway to Globalization). 1995;2:1882-6. doi:10.1109/ICC.1995.524287. 